\title{
Community Based Intervention for Tobacco Cessation: A Pilot Study Experience, North East India
}

\author{
Srabana Misra Bhagabaty ${ }^{*}$, Amal Chandra Kataki², Manoj Kalita ${ }^{3}$, Shekhar \\ Salkar $^{4}$
}

\begin{abstract}
Background: North East India has a high prevalence of tobacco consumption, but only few individualsseek help for tobacco cessation. Impact of community based tobacco cessation intervention in this part needs more research. Materials and Methods: Retrospective analysis was done on the dataset from a community-based tobacco cessation intervention pilot project conducted in Guwahati metro during 2009-10. Subjects, both male and female tobacco users, age $>15$ years, permanent residents of these blocks giving consent were included in the study. Results: The sample was 800 tobacco users, of whom $25 \%$ visited any health care provider during last 12 months and 3\% received tobacco cessation advice. An 18\% quit rate was observed at six weeks follow up, more than the National average, with a $47 \%$ quit rate at eight months, while $52 \%$ of subjects reduced use. Conclusions: Higher tobacco quit rate and reduced tobacco use, no loss to follow up and negligible relapse was observed with this community based intervention design. Such designs should be given more emphasis for implementation in specified communities with very high tobacco consumption rates, cultural acceptance of tobacco and less motivation towards quitting.
\end{abstract}

Keywords: Tobacco cessation - community - intervention - tobacco - North East India

Asian Pac J Cancer Prev, 16 (2), 811-814

\section{Introduction}

Tobacco is a burning issue. India is the second largest consumer and third largest producer of tobacco (Jhanjee, 2011). Tobacco related diseases are preventable causes of death to a large extent yet these diseases killing people worldwide. Around the world, nearly 5.4 million people every year die from lung cancer, heart disease and other illnesses. If current trends continue tobacco will account for $13 \%$ of all deaths by 2020 . Nearly 900,000 people die every year in India due to diseases attributed to tobacco (Jayakrishnan et al., 2011) .Tobacco is a well known risk factor of cancer. The International Agency for Research on Cancer (IARC) Monograph states that tobacco smoking is the major cause of lung cancer (all types) and is majorly associated with oral cancer, cancers of the oropharynx and hypopharynx, oesophagus, stomach, nasopharynx (IARC, 1987). More than fifty percent of all cancer cases reported in NE-India was tobacco related cancers (NCRP, 2013). Cardiovascular and respiratory diseases are as well tobacco related.

In India, head and neck cancers (HNCA) account for $30-40 \%$ cancers at all sites, out of which $9.4 \%$ being oral cancers which is sixth common cause of death in males and seventh in females. Oral cancer is one of the most common cancers in the world, commonest in India, Bangladesh,
Srilanka and Pakistan. In North-east India, incidence of tobacco related oral cancers is about 33\% (Bhattacharjee et al., 2006). The relative proportion tobacco related cancer of kamrup urban of Assam state shows that $6.47 \%$ of male and $2.87 \%$ of females had oral cancer (NCCP, 2005). Out of the total cancer cases registered at Guwahati Hospital Based Cancer Registry during 2011-12 shows that 64.3\% cancers in males and $28.2 \%$ cancers in females were Tobacco related (HBCR Guwahati, 2012).

More than one third (35\%) of adults in India use tobacco in some form and $84 \%$ of the users use tobacco every day (Mini et al., 2014). About two in five adults from rural and one in four from urban areas use tobacco. Prevalence of tobacco use is $48 \%$ in males in comparison to $20 \%$ amongst female. If we compare with the national scenario of tobacco use, current tobacco use pattern is really atrocious in the entire North Eastern Region, where Assam (39\%), Sikkim (42\%), Arunachal Pradesh (48\%), Manipur (54\%), Meghalaya (54\%), Tripura (56\%), Nagaland (56\%), exceeds far from the National average and Mizoram with $67 \%$, tops the list amongst all the union territories covered by Global Adult Tobacco Survey (GATS , 2010). No doubt, as a whole India has a very high rate of tobacco use throughout but in the North East region of India shows the exigency to the need to do something intensive towards effective tobacco control in this region. 
The cultural acceptance of tobacco use makes the picture even worse. Tobacco is consumed in every possible way in this part of the country; it is smoked, chewed and even used as drink Tibur, as a tradition (Malakar et al., 2012; Sharma et al., 2013).

Current users of tobacco show variations with education level. In India literacy is inversely proportional to tobacco use. Rural vs. urban percentages of current tobacco users being $25 \%$ and $38 \%$ respectively (Jayakrishnan et al., 2011). Tobacco use differs from place to place from population to populations with pockets where consumption is alarmingly high and needs immediate attention.

Probable consumers of tobacco in near future may add to the demand pool of tobacco products by adding already existing tobacco demands. This may make the control of production, sale and distribution of tobacco even more cumbersome. Pools of both current and potential users of tobacco should be addressed for successful tobacco control.

To decrease the demand, users of tobacco have to quit the habit. India reports that only $38.4 \%$ of current tobacco users above 15 years of age made a quit attempt in the last 12 months. These figures are much lesser in the North Eastern states. Less than half of the current users of tobacco in India visited any health care provider during last 12 months out of which only $46.3 \%$ of current smokers and $26.7 \%$ of current chewers got any advice to quit tobacco by any health care provider. In North East these figures are even lower and only $24.7 \%$ current smokeless tobacco users and $41.6 \%$ current smokers got any advice to quit the tobacco habit by any health care provider (GATS, 2010).

Research question: As in India specially in the North Eastern States, health seeking behavior amongst the people is still far behind the other developed nations, the possibility of coming to a health care provider or a clinic for the sole reason of quitting tobacco is still a question. Here the researchers tried to analyze if there could be any affirmative impact if care providers go out to the community to provide cessation help. Some of such community based tobacco cessation intervention studies has been conducted outside India, and few studies in India. But In North East India with a high tobacco use prevalence and low motivation towards quitting and health seeking behavior, such intervention design should be tried to see the feasibility. This is a first such kind of pilot study in this region.

Objectives of this study: $i$ ) To find out the practicability of providing tobacco cessation intervention at community level. ii) To analyze the success of tobacco cessation services extended to the community level. iii) To see the prospect of implementation of Community based tobacco cessation intervention at large scale and to suggest recommendations for better tobacco cessation service

\section{Materials and Methods}

Data obtained from a community based tobacco cessation intervention study at Guwahati city in the year 2009-2010. It was carried out in South, East, West and Central urban blocks of the city . Four Medical Social
Workers (MSW) carried out the community based intervention and follow up. Each of the MSW was allotted with one zone. The MSWs were trained up on the tobacco scenario, tobacco hazards and counseling and basic knowledge on tobacco control and cessation services. These MSW surveyed their respective areas door to door starting from a prominent landmark till they found the required no. of study subjects fulfilling the inclusion and exclusion criteria. The inclusion criteria for the study subjects being, both male and female users of tobacco of age $>15$ years and permanent residents of study areas. The exclusion criteria were non users of tobacco, tobacco users of age less than 15 years, not willing to get tobacco cessation intervention, not permanent residents of the study areas.

Sample for the study was 800. Each MSW registered 200 study subjects. Study duration was 12 months. The first two months were used for, door to door survey in the intervention areas and registration of the subjects. A predesigned proforma was filled up during registration of the study subjects. Detailed history of the tobacco use pattern along with addiction and motivation level was included in the proforma. Proforma contained follow up status estimation questions which was filled up during each follow up.

During registration, IEC materials on tobacco hazards were offered to the subjects and sensitized them on the tobacco hazards. A date and time for counseling was fixed according to the convenience of the subjects a keeping in mind time frame of the study .

The mode of intervention was tobacco cessation counseling offered by the MSW at the community setting at homes of the study subjects.

After counseling, the subjects were followed up for eight months. Follow up was done by direct contact at 2 weeks, 4 weeks, 6 weeks, 2 months, 3 months, 4 months, 6 months, 8 months. When contacted for follow up the study subjects if needed re counseling was given to them.

\section{Results}

The survey covered 750 households with $87 \%$ of these househols with a neuclear family and total numbers of persons in the age group $>15$ years and parmanent residents of these areas were 2250 out of which 809 were current tobacco users . 9 of these 809 did not want to get intervened. 800 study subjects were registered and intervened for tobacco cessation. This study population showed current tobacco use of $36 \% .81 \%$ of the study subjects were males and $19 \%$ females. $78 \%$ of the study subjects were in the age group of 20-39 years, $18 \%$ in $40-59$ years while $3 \%$ in the age group of $10-19$ years $(<$ 15 years were not included in the study and not counted) and $1 \%$ above 60 years. (Figure 1)

Before registering the subjects were enquired if they visited any health care service for any reason and how many of them actually got any advice to quit the tobacco use habit. It was found that only $1 / 4$ th of the subjects (current tobacco users) visited any health care provider during last 12 months. $15 \%$ of current tobacco users (both smoking and smokeless type) got any kind of advice to 


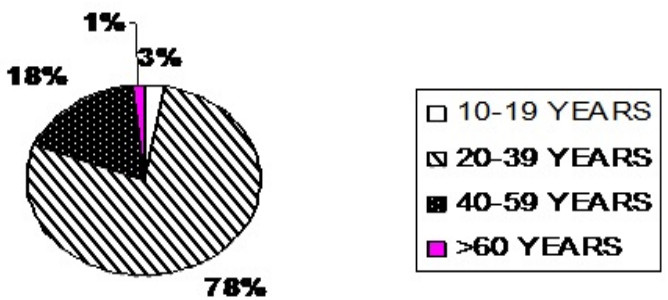

Figure 1. Age Distribution of the Subjects

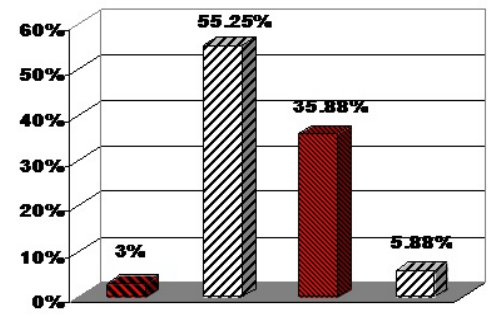

\section{ब $<2000$} $\square 2001-5000$ 5001-10,000 $\square>10,000$

Figure 2. Distribution of the Subjects According to Income

Table 1. Results of Follow Up at Eight Months Post Intervention

\begin{tabular}{lrr}
\hline Outcome & \multicolumn{2}{c}{ Number $(\%)$} \\
\hline Quit Tobacco & 376 & $(47 \%)$ \\
Reduced tobacco use (More than 50\%) & 415 & $(52 \%)$ \\
No change & 0 & $(0 \%)$ \\
Loss to follow up & 0 & $(0 \%)$ \\
Relapse & 6 & $(1 \%)$ \\
Total & $800(100 \%)$ \\
\hline
\end{tabular}

quit the tobacco use habit. $10 \%$ of the study subjects were illiterate, $18 \%$ completed their primary school education, $30 \%$ middle school, $15 \%$ high school, $22 \%$ higher secondary and $15 \%$ completed educaion level of degree and above (Figure 2). $3 \%$ were in the income group less than rupees 2000 per month, $55 \%$ fall in the income group of between rupees 2000 and 5000 per month, 36\% had per month income between rupees 5000 and 1000, and 6\% showed income 10000 and above per month.

After couselling each subject was followed up. At six weeks post intervention follow up 18 out of 100 users have quitted the tobacco use habit at the time of follow up. A very high quit rate was observed (Table 1) at eight months post intervention follow up which was $47 \%$ with only tobacco cessation counseling intervention and regular scheduled follow up visits.

\section{Discussion}

Current tobacco use pattern in the study pupulation $(36 \%)$ tallies with national $(34.6 \%)$ and state level $(39.3 \%)$ current tobacco use pattern (GATS, 2010). This cross section of the community somewhere reflected inclination towards tobacco use in the younger age group in comparison to the National level. Maximum numbers of the users in the study population was in the age group of $15-39$ years which is $81 \%(648)$. Whereas in national context, age group distribution of tobacco users showed maximum numbers in the age group above 65years(48\%) followed by $45-64$ years $(47 \%), 25-44$ years $(37 \%)$ and $15-24$ years $(18 \%)$ (GATS, 2010). Only $25 \%$ of the study sample visited any health care provider during last 12 months and only 3\% received any kind of tobacco cessation advice. This is much lower than the national health seeking behavior where less than half of the current users of tobacco visited any health professional. If we consider the national scenario, $46.3 \%$ of current smokers and $26.7 \%$ of current chewers got any advice to quit tobacco by any Health Care Provider. The picture is even worst in north east only where visit to any health care provider is much lower and only $24.7 \%$ current smokeless tobacco users and $41.6 \%$ current smokers got any advice to quit the tobacco habit by any health care provider. The picture was even menacing amongst the study subjects where only $15 \%$ of current tobacco users (either smoking or smokeless type) got any kind of advice to quit the tobacco use habit. With such low level of health seeking behavior and the low possibility of getting any cessation advise as well as availability of only few tobacco cessation centers in this region, reaching out to the community to provide them tobacco cessation help may be of great help.

Awareness about the ill effects of tobacco and the approch to any health facility is higher in the well educated strata of the community hence health seeking behaviour of people increases with level of education and socioeconomic status. In the study $10 \%$ subjects were completely illiterate. Morever majority of the subjects were in the lower family income group of rupees 20015000 per month. When study areas were approched for community based intervention, the whole community got access to the service regardless their educational or economic background. So commuty interventions can address needs of the needy where cessation help goes to their doorsteps and here by by passing all the barriers that limits the mass from seeking tobacco cessatin help.

Tobacco cessation centers (TCC) from India, have reported overall quit rates of around $16 \%$ at six weeks post intervention. The addition of pharmacological adjuncts improves the quit rates. Agents like the anti-depressants, bupropions have been shown to increase the quit rates in the treatment of nicotine dependence by approximately 1.5 to two times, irrespective of the settings. Combined with behavioral interventions they produce quit rate up to $35 \%$ (TCC India) (NCCP, 2005).

The six weeks follow up of the intervened subjects under study showed still a higher rate (18\%) of quitting in comparison to the national average (16\%) as reported by TCCs of India.. The lost to follow up is zero at six weeks post intervention follow up as well as at the eight month post intervention. In clinic based cessation interventions for tobacco control, either the registered users themselves have to visit to the centre for follow up or they can be contacted over phone for follow up. But as per our clinic based experiences from TCC Guwahati, it was obvious that most people either don't revisit for follow up or could not be contacted over phone due to either nonavailability of phone numbers, wrong phone numbers, non receipt of phone calls, out of reach phone numbers or switched off mobile, non delivered, on replying of phone message . By the end of one year only few numbers of 
cessation help seekers at the clinic were traceable where as all of the community based subjects getting cessation interventions could be followed up without miss as they could be followed up by visiting their homes. The results were very much obvious reflected in their high quit rate at the end of eight month post intervention.

It is really important to follow up the subjects who were under tobacco influence for a better quit rate and who are in the maintenance phase to maintain the status. Those who are in contemplation and preparation stage also can go into the action phase through scheduled follow up visits and if needed giving re-counseling during these follow up visits. Such vigorous follow up is possible only by personal contact with the subjects, but in clinic based services it is not feasible. Less follow up means more relapse.

This study observed that with the unmissed timely follow up the quit rate was added and maintained to give a high quit rate of $47 \%$ at the end of eight months post intervention (with only behavioral counseling) and 52\% of the study subjects were in the stage of reduced use and regular scheduled follow up and re counseling have all the bright possibility of towing them towards quitting the habit.

\section{Conclusion and recommendation}

Community based tobacco cessation intervention is a good model for tobacco cessation where only with cessation counseling as an intervention tool, we observed a very high quit rate of $47 \%$ at eight months post intervention follow up. This quit rate can be even further increased by adding pharmacological adjuncts along with counseling. Lost to follow up was zero and relapse rate was negligible. So if we opt for a better follow up and a better quit rate in a defined population, the community based tobacco cessation interventions could be an answer.

Such vigorous community based tobacco cessation intervention designs should there for be given more emphasis on for implementation in a specified community with a very high tobacco consumption rate, cultural acceptance of tobacco and less motivation towards quitting to get: $i$ ) Better results towards awareness generation about tobacco hazards and benefits of quitting. ii) To address needs of the needy bypassing all the barriers that limits the mass from seeking tobacco cessatin help. iii) To attain high tobacco quit rate and lesser lost to follow up and close monitoring of maintenance of tobacco cessation

\section{References}

Bhattacharjee A, Chakraborty A, Purkaystha P (2006). Prevalence of head and neck cancers in the North East- an Institutional study. Indian J Otolaryngol Head Neck Surg, $\mathbf{5 8}, 15-9$.

Global Adult Tobacco Survey (GATS): India Report 2009-2010 (2010). Ministry of Health and Family Welfare, Government of India, 1-36.

Hospital Based Cancer Registry,Guwahati (2012), National cancer registry programme, Indian council of medical research. BBCI annual report for the year of 2011-12

International Agency for Research on Cancer (IARC) (1987). IARC monographs on the evaluation of the carcinogenic risks to humans, supplement 7 . overall evaluations of carcinogenicity: an updating of IARC monographs volumes 1.42. Lyon: IARC Press, 357-61

Jayakrishnan R, Mathew A, Uutela A, et al (2011). A community based smoking cessation intervention trial for rural Kerala, India-preliminary results. Asian Pac J Cancer Prev, 12, 3191-5.

Jhanjee S (2011). Tobacco control in India-Where are we now?. Delhi Psychiatry J, 14, 26-32.

Malakar M, Devi KR, Phukan RK, et al (2012). Genetic polymorphism of glutathione S-transferases M1 and T1, tobacco habits and risk of stomach cancer in Mizoram, India. Asian Pac J Cancer Prev, 13, 4725-32.

Manuals for training in cancer control: Manual for tobacco cessation, (NCCP) National cancer control programme (2005): Directorate general of health services: Ministry of Health and Family Welfare, Government of India.

Mini GK, Sarma PS, Thankappan KR (2014). Pattern of tobacco use and its correlates among older adults in India. Asian Pac J Cancer Prev, 15, 6195-8.

National Cancer Registry Programme (ICMR) (2013). Threeyear report of the population based cancer registries: 20092011. Bangalore, India

Sharma JD, Kalita M, Nirmolia T et al (2014). Cancer: scenario and relationship of different geographical areas of the globe with special reference to North East-India. Asian Pac J Cancer Prev, 15, 3721-9. 